\title{
Análise comparativa da abundância do coral invasor Tubastraea spp. (Cnidaria, Anthozoa) em substratos naturais e artificiais na Ilha Grande, Rio de Janeiro, Brasil
}

\author{
Tárcio Santos Mangelli1 \& Joel Christopher Creed $^{2}$
}

\begin{abstract}
1. Pós-Graduação em Ciências Ambientais, Centro Universitário de Barra Mansa (Cicuta). Rua 35, 714, Fazenda Santa Cecília, 27261-140 Barra Mansa, RJ. (tarciosm@yahoo.com.br)
2. Laboratório de Ecologia Marinha Bêntica, Instituto de Biologia Roberto Alcântara Gomes, Universidade do Estado do Rio de Janeiro. Rua São Francisco Xavier, 524, PHLC Sala 220, 20550-900 Rio de Janeiro, RJ. (jcreed@uerj.br)
\end{abstract}

\begin{abstract}
Comparative analysis of the invasive coral Tubastraea spp. (Cnidaria, Anthozoa) on natural and artificial substrates at Ilha Grande, Rio de Janeiro, Brazil. Exotic sun corals or cup corals (Tubastraea coccinea Lesson, 1829 and T. tagusensis Wells, 1982) have invaded sessile biological communities on rocky shores at Ilha Grande Bay, Rio de Janeiro, Brazil. Artificial structures (piers, docks or decks) were selected at four sites in Ilha Grande and compared with natural substrates on the adjacent rocky shore by quantifying the density of each species of Tubastraea, as well as the percentage coverage of the benthic biota. The overall average density of Tubastraea tagusensis was 80.3 individuals. $\mathrm{m}^{-2}$, about double that of T. coccinea. Both invasive species modified the structure of the invaded communities as relative abundance and richness of the species changed due to their presence. Tubastraea coccinea was more abundant on artificial substrates than on natural ones but no difference was detected in the abundance of Tubastraea tagusensis between substrates. The abundance of both species also varied with depth and site and a positive relation between artificial substratum age and abundance of the corals was observed. Apparently T. tagusensis is competitively superior to $T$. coccinea with a greater ability to occupy/invade native communities on natural substrates. Both species showed high competitive potential and can use artificial substrates to start to establish in new areas.
\end{abstract}

KEYWORDS. Biological invasion, competition, community, density, Scleratinia.

RESUMO. Os corais exóticos coral-sol (Tubastraea coccinea Lesson, 1829 e T. tagusensis Wells, 1982) invadiram as comunidades biológicas sésseis dos costões rochosos da Baía da Ilha Grande, Rio de Janeiro, Brasil. Estruturas artificiais (piers, decks ou cais) foram selecionadas em quatro locais na Ilha Grande, e comparadas com substratos naturais adjacentes através de quantificação da densidade de cada espécie de Tubastraea, além da porcentagem de cobertura da biota bêntica. A densidade média geral de Tubastraea tagusensis foi de 80,3 indivíduos.m² ${ }^{2}$, aproximadamente o dobro encontrado para $T$. coccinea. Tendo em vista que sua presença alterou as abundâncias relativas e a riqueza de espécies, ambas as espécies invasoras modificaram a estrutura das comunidades invadidas. Tubastraea coccinea foi mais abundante em substratos artificiais do que naturais, porém, não foi detectada diferença na abundância de Tubastraea tagusensis entre substratos. A abundância de ambas as espécies variou com a profundidade e o local, e houve uma relação positiva entre a idade do substrato artificial e a abundância dos corais. Aparentemente T. tagusensis é competitivamente superior à T. coccinea, com uma inserção maior nas comunidades nativas em substratos naturais. Ambas as espécies tem grande potencial competitivo e podem utilizar substratos artificiais para iniciar seu estabelecimento em novos locais.

PALAVRAS-CHAVE. Bioinvasão, competição, comunidade, densidade, Scleractinia.

Espécies exóticas, ou não nativas, são aquelas encontradas além de sua área de distribuição geográfica historicamente conhecida. Chegam a novos locais através de vetores criados pela ação humana, em um ou mais eventos de introdução. Bioinvasores são espécies exóticas que aumentam sua densidade e abrangência geográfica substancialmente após a chegada, assim modificando com sua presença as comunidades biológicas receptoras e criando novas interações ecológicas. Como espécies nativas são adaptadas ao ambiente onde se encontram ao longo de milhares de anos, uma bioinvasora se estabelecerá em uma comunidade nativa, provavelmente, em três cenários: 1) um ambiente abiótico receptor muito similar ao ambiente de origem do bioinvasor; 2) o bioinvasor ser altamente competitivo perante as espécies na comunidade receptora; ou 3) o ambiente abiótico e consequentemente comunidades receptoras serem perturbadas por alterações antrópicas (TYRRELL \& BYERS, 2007). O último mecanismo, a modificação do regime de seleção, tem sido considerado responsável por explicar a observação de que há correlações entre grau de perturbação e bioinvasão (HoBBS \& HuENNEKE, 1992).
Para organismos marinhos sésseis qualquer substrato é um habitat em potencial, e substratos artificiais representam um ambiente modificado pelo homem que, em termos evolutivos, é tanto estranho para bioinvasores quanto para organismos nativos (BYers, 2002; TyRreLL \& BYERs, 2007). Estudos recentes têm observado que substratos artificiais podem abrigar comunidades diferentes aos substratos naturais próximos (BULLERI \& ChapMan, 2004). Tyrrell \& Byers (2007) argumentaram que bioinvasores devem ser mais abundantes em substratos artificiais do que em naturais, pois as espécies nativas selecionam preferencialmente substratos locais naturais e ainda devem ser competitivamente superiores em tais substratos quando comparados com bioinvasores. Entretanto, em estudos recentes, as evidências são contraditórias: BULLERI \& AIROLDI (2005), WASSON el al. (2005) e TYRrell \& Byers (2007) encontraram maior abundância de bioinvasores em substratos artificiais enquanto Creed \& Paula (2007) e Cangussu et al. (2010) não obtiveram tal padrão.

Corais do gênero Tubastraea (Cnidaria, Anthozoa, Scleractinia, Dendrophylliidae) são azooxantelados que crescem em águas rasas, em recifes de coral e costões 
rochosos tropicais (CAIRNS, 2000). Nativos dos oceanos Pacífico e Índico, hoje algumas espécies são consideradas cosmopolitas, sendo amplamente distribuídas em águas tropicais do Atlântico, Pacífico e Índico devido ao processo de bioinvasão (PAULA \& CREed, 2004).

Duas espécies são consideradas invasoras: Tubastraea coccinea Lesson, 1829 e Tubastraea tagusensis Wells, 1982. O primeiro registro de uma espécie de Tubastraea como bioinvasor foi em 1943 quando T. coccinea foi registrada no Caribe (CAIRns, 2000). Hoje se encontra distribuída em várias regiões do Caribe e Golfo do México, tanto em substratos naturais quanto em substratos artificiais, principalmente naqueles associados à indústria petrolífera, tais como plataformas de petróleo e gás, monobóias, entre outros (CAIRNS, 1994, 2000; Fenner, 2001; Fenner \& Banks, 2004; SAmmarco et al., 2004). Em 2010, outra espécie deste gênero, T. micranthus (Ehrenberg, 1834), foi registrada como espécie exótica com potencial invasor em uma plataforma de petróleo no Golfo do México (Sammarco et al., 2010).

No Brasil, o primeiro registro de Tubastraea foi em uma plataforma de petróleo na Bacia de Campos, RJ (PAula \& CReed, 2005). A bioinvasão nos costões rochosos por Tubastraea iniciou em Ilha Grande, RJ, na década de 1980 (CASTRO \& Pires, 2001), embora apenas em 2004 tenham sido identificadas duas espécies do gênero na região: T. coccinea e T. tagusensis, esta última com distribuição nativa no Arquipélago de Galápagos, Equador (Paula \& Creed, 2004). Também foi registrada a bioinvasão de $T$. coccinea em Arraial de Cabo, RJ (FERREIRA, 2003).

Atualmente, T. coccinea e T. tagusensis são encontradas nos costões rochosos na Baía da Ilha Grande, onde ocupam e podem dominar áreas do infralitoral raso (Paula \& CReed, 2005). O estabelecimento e a expansão geográfica destes corais nesta região têm resultado em mudanças nas comunidades bentônicas e danos ao coral nativo e endêmico no Brasil Mussismilia hispida (Verrill, 1902) (Creed, 2006; Creed \& Paula, 2007).

Devido às atividades de expansão imobiliária e navegação, a Baía da Ilha Grande vem recebendo substratos artificiais que são compostos de diversos materiais, tais como: estruturas de cimento, pneus velhos, pilares de piers em PVC, decks de madeira, entre outros. De acordo com SAmmarco et al. (2004), substratos artificiais podem oferecer a Tubastraea e a outros corais a oportunidade de aumentar sua abrangência geográfica através de "trampolins" em colonização (chamada "leap-frogging" ou "stepping stones") (LiRA et al., 2010). É um mecanismo muito bem documentado em bioinvasões (ELTON, 1958) e neste caso a introdução de um novo habitat facilita a expansão geográfica do bioinvasor (efetivamente criando um beachhead, sensu SimBerLoff (1997), para passagem posterior aos substratos naturais adjacentes). Ainda, como Tubastraea spp. foi introduzido no Brasil através de bioincrustação em substratos artificiais (plataformas de petróleo e gás - Paula \& CReEd, 2005) é provável que estas espécies exóticas invasoras sofram forte pressão seletiva à utilização de tais substratos durante transporte pelo vetor.

O objetivo deste estudo foi utilizar o coral-sol (Tubastraea spp.) para testar a hipótese que substratos artificiais devem ter maior abundância de Tubastraea spp. quando comparados com substratos naturais adjacentes.

\section{MATERIAL E MÉTODOS}

A Ilha Grande possui uma área de $193 \mathrm{~km}^{2}$ e está localizada no município de Angra dos Reis, entre $23^{\circ} 05$ - $23^{\circ} 14 \mathrm{~S}$ e $44^{\circ} 05-44^{\circ} 23 \mathrm{~W}$, região sudeste do estado do Rio de Janeiro, Brasil (Fig. 1). As águas adjacentes possuem um intenso tráfego de embarcações de grande porte devido à localização de um porto, um estaleiro, terminais de minério de ferro, petróleo e marinas, sofrendo consequentemente impactos causados pela inserção de estruturas artificiais nos costões rochosos (Creed \& Oliveira, 2007).

Foram selecionadas quatro regiões na Ilha Grande ou em seus arredores onde houve registro prévio do coral-sol (CREED et al., 2008): Lagoa Azul $\left(23^{\circ} 05,715^{\prime} \mathrm{S}, \quad 44^{\circ} 14,672^{\prime} \mathrm{W}\right)$, Ilha Aroeira $\left(23^{\circ} 04,662^{\prime} \mathrm{S}, \quad 44^{\circ} 14,401^{\prime} \mathrm{W}\right)$, Enseada Araçatiba $\left(23^{\circ} 09,322^{\prime} \mathrm{S}, 44^{\circ} 19,805^{\prime} \mathrm{W}\right)$ e Feiticeira $\left(23^{\circ} 07,095^{\prime} \mathrm{S}\right.$, $44^{\circ} 10,903^{\prime}$ 'W). Em cada região foram selecionadas entre um e três locais com estruturas artificiais distintas, gerando oito locais no total.

Através de mergulho autônomo foram quantificadas a porcentagem de cobertura e a densidade de cada espécie de Tubastraea, além da porcentagem de cobertura dos outros organismos ou grupos funcionais possíveis de serem identificados in situ. Para isso, foram colocadas três transecções verticais em substratos artificiais posicionados aleatoriamente em cada estrutura (=local). As amostragens foram feitas em quadrados $(20 \times 20 \mathrm{~cm})$ dispostos a cada $50 \mathrm{~cm}$ até a base do substrato. Foram utilizados apenas substratos de orientação vertical. Em substratos naturais adjacentes, para cada local foram dispostos transecções $(n=3)$ em posições aleatórias, usando o mesmo método de amostragem e quantificação. Quando não houve disponibilidade de substrato vertical em determinado ponto na transecção, foi amostrado o primeiro substrato vertical encontrado à direita da transecção seguindo a mesma linha de profundidade, sendo desta forma também, apenas substratos verticais amostrados no costão rochoso. Como a profundidade máxima de substratos artificiais variou entre locais e até entre transeções (Lagoa Azul 1: 1,6-3,5 m; Lagoa Azul 2: 2,5 m; Lagoa Azul 3: 2,5-3 m; Aroeira: 0,8 m; Araçatiba 1: 2-3,5 m; Araçatiba 2: 1,5-3,5 m; Araçatiba 3: 2-2,5 


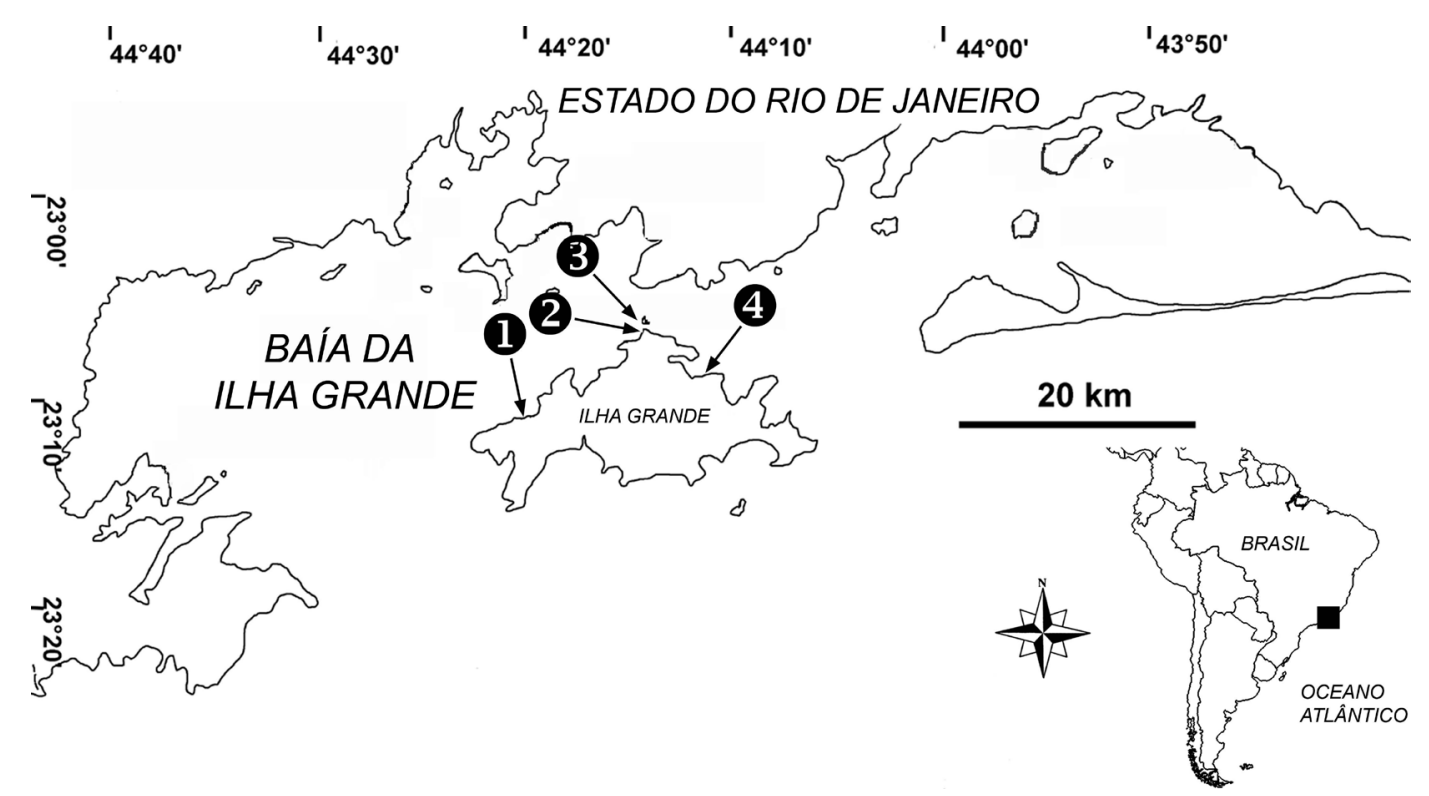

Fig. 1. Localização dos pontos de amostragem, Ilha Grande e arredores, estado do Rio de Janeiro, Brasil: 1, Araçatiba; 2, Lagoa Azul; 3, Aroeira; 4, Feiticeira.

m; Feiticeira: 3-3,5 m), estas mesmas profundidades foram utilizadas como limites para amostrar os substratos naturais.

Além da comunidade biológica, foram coletados dados sobre a composição e idade (tempo na água) dos substratos artificiais através de observação in situ e questionário aos residentes, respectivamente. A relação entre a idade de substrato artificial (anos) e a densidade média de colônias de Tubastraea spp. foi analisada por Regressões Lineares Simples.

Para análise dos dados utilizou-se a análise de covariância (ANCOVA, SPSS v 19), tendo como fatores o local e o substrato e como co-variável a profundidade. A Análise de Similaridade (ANOSIM) foi aplicada para testar a significância de separação dos grupos considerados neste estudo em relação ao fator tipo de substrato e para testar se o grupo de transecções (comunidades) com presença de Tubastraea coccinea ou T. tagusensis diferiram do grupo de transeções sem o coral-sol. Para verificar a similaridade entre as comunidades, foi utilizada a Análise de Escalonamento Multidimensional (EMD) baseada no Coeficiente de Similaridade de Bray-Curtis em dados transformados em raiz quadrada e padronizados (Primer 5, Primer E Ltd.).

\section{RESULTADOS}

Foram identificados 23 táxons/grupos funcionais, sendo 15 invertebrados e 8 algas (Tab. I). As algas tufo foram as mais abundantes, tanto em substrato artificial quanto em natural. Seis táxons foram encontrados exclusivamente em substrato natural e cinco apenas em substrato artificial. Palythoa caribaeorum Duchassaing \& Michelotti, 1860 foi o segundo táxon mais abundante em substrato natural (11\% de cobertura), mas não foi detectado em substratos artificiais. $\mathrm{O}$ coral exótico
Tubastraea coccinea foi o quinto mais abundante $(3,9 \%)$ em substratos artificiais e o oitavo em substratos naturais $(1,1 \%)$, enquanto Tubastraea tagusensis foi o sétimo mais abundante em ambos os substratos $(2,5 \%$ em artificiais e $1,2 \%$ em naturais).

A densidade de $T$. coccinea variou entre locais e substratos embora não tenha havido interação significativa entre estes fatores e a profundidade não foi uma covariável significante (ANCOVA, Tab. II). Apesar disso a densidade de Tubastraea coccinea ficou concentrada entre 1 e $2 \mathrm{~m}$ (Fig. 2) e $T$. coccinea foi significativamente mais abundante em substratos artificiais do que em naturais (Tabela II; Fig. 4). Em contraste, T. tagusensis não mostrou uma diferença significativa em densidade entre substratos artificiais e naturais ou para a interação dos fatores substrato e local (Tab. III; Fig. 4) apenas variando significativamente entre locais (Tabela III; Figs 5, 6). Profundidade foi uma covariável significativa e Tubastraea tagusensis apresentou densidade mais equilibrada entre as profundidades (Fig. 3). A densidade média de Tubastraea spp. em substratos artificiais foi de 128 indivíduos. $\mathrm{m}^{-2}$ e em substratos naturais foi de 82 indivíduos. $\mathrm{m}^{-2}$.

As duas espécies de Tubastraea foram encontradas em todos os locais estudados com a exceção da Feiticeira, onde não foi registrada T. tagusensis (Figs 5, 6). A densidade média geral de $T$. tagusensis nos locais estudados na Ilha Grande (80,3 indivíduos. $\left.\mathrm{m}^{-2}\right)$ foi aproximadamente o dobro de $T$. coccinea $(41,1$ indivíduos. $\mathrm{m}^{-2}$ ). A maior densidade de $T$. coccinea foi observada na Lagoa Azul I (108 indivíduos.m-2) e a menor foi na Feiticeira (1,1 indivíduos. $\mathrm{m}^{-2}$ ) (Fig. 5). A maior densidade de T. tagusensis foi observada na Aroeira (340 indivíduos. $\mathrm{m}^{-2}$ ) (Fig. 6).

$\mathrm{Na}$ Ilha Grande houve uma relação positiva 
e significativa entre a idade do substrato artificial e a densidade de colônias de Tubastraea coccinea (Densidade $=3,5 \times$ Ano $-1,73 ; n=8, R^{2}=67 \%, p=0,013$ ), embora não houve relação significativa para T. tagusensis $\left(\right.$ Densidade $=1,46 \times$ Ano $+17,9 ; \mathrm{n}=8, \mathrm{R}^{2}=10,8 \%$, $\mathrm{p}=0,427)$.

A ordenação das comunidades estudadas revelou uma separação por tipo de substrato principalmente no eixo principal (Fig. 7) que foi significativa (ANOSIM $R=0,148, p=0,002$ ). A presença de $T$. tagusensis (Fig. 8) e T. coccinea (Fig. 9) foi parcialmente responsável na ordenação, sendo que cada espécie mostrou sua presença em agrupamento separado (ANOSIM: grupo com presença vs. ausência de $T$. tagusensis: $R=0,245$, $p=0,001$; grupo com presença vs. ausência de $T$. coccinea: $R=0,143, p=0,002)$.

Tab. I. Táxons/grupos funcionais encontrados em substratos naturais e artificiais na Ilha Grande, estado do Rio de Janeiro, Brasil.

\begin{tabular}{|c|c|c|}
\hline \multirow[t]{2}{*}{ Taxon/Grupo } & \multicolumn{2}{|c|}{$\begin{array}{l}\text { Cobertura média } \\
\% \text { (erro padrão) }\end{array}$} \\
\hline & Artificial & Natural \\
\hline \multicolumn{3}{|l|}{ Arthropoda, Maxillopoda, Sessilia } \\
\hline Balanidae & $10,31(1,51)$ & $8,07(1,49)$ \\
\hline \multicolumn{3}{|l|}{ Bryozoa, Stenolaemata, Cheilostomata } \\
\hline Schizoporella sp. & $1,26(0,52)$ & $0(0)$ \\
\hline Bryozoa sp. 1 & $0,11(0,11)$ & $0,42(0,31)$ \\
\hline Chordata, Ascidiacea & $0,42(0,15)$ & $0,65(0,25)$ \\
\hline \multicolumn{3}{|l|}{ Cnidaria, Anthozoa, Actiniaria } \\
\hline Bunodosoma caissarum Corrêa, 1964 & $0,10(0,08)$ & $0(0)$ \\
\hline \multicolumn{3}{|l|}{ Gorgonacea } \\
\hline Carijoa riisei Duchassaing \& Michelotti, 1860 & $2,65(0,98)$ & $0,18(0,18)$ \\
\hline \multicolumn{3}{|l|}{ Scleractinia } \\
\hline Tubastraea coccinea Lesson, 1829 & $3,87(1,11)$ & $1,14(0,57)$ \\
\hline Tubastraea tagusensis Wells, 1982 & $2,46(0,82)$ & $1,25(0,48)$ \\
\hline Mussismilia hispida Verril, 1902 & $0,05(0,05)$ & $0,18(0,12)$ \\
\hline Madracis decactis (Lyman, 1859) & $0(0)$ & $0,05(0,05)$ \\
\hline \multicolumn{3}{|l|}{ Zoanthidea } \\
\hline Palythoa caribaeorum Duchassaing \& Michelotti, 1860 & $0(0)$ & $11,30(2,26)$ \\
\hline Zoanthus sociatus Ellis, 1767 & $0(0)$ & $0,09(0,09)$ \\
\hline \multicolumn{3}{|l|}{ Mollusca, Bivalvia, Mytiloida } \\
\hline Perna perna (Linnaeus, 1758) & $0,06(0,06)$ & $0(0)$ \\
\hline \multicolumn{3}{|l|}{ Pterioida } \\
\hline Ostrea $\mathrm{sp}$. & $0,62(0,26)$ & $0(0)$ \\
\hline Porifera, Demospongiae & $18,11(3,94)$ & $7,30(1,77)$ \\
\hline \multicolumn{3}{|l|}{ Chlorophyta, Bryopsidophyceae } \\
\hline Caulerpa racemosa (Forsskål) J. Agardh & $5,05(2,11)$ & $3,32(1,96)$ \\
\hline Codium intertextum Collins \& Hervey & $0(0)$ & $0,03(0,03)$ \\
\hline \multicolumn{3}{|l|}{ Phaeophyta, Phaeophyceae, Fucales } \\
\hline Sargassum $\mathrm{sp}$. & $1,05(0,79)$ & $0,67(0,43)$ \\
\hline \multicolumn{3}{|l|}{ Dictyotales } \\
\hline Padina sp. & $0(0)$ & $0,09(0,09)$ \\
\hline \multicolumn{3}{|l|}{ Rhodophyta, Florideophyceae, Bonnemaisoniales } \\
\hline Asparagopsis taxiformis (Delile) Trevisan de Saint-Léon & $0(0)$ & $0,29(0,22)$ \\
\hline \multicolumn{3}{|l|}{ Rhodophyceae, Ceramiales } \\
\hline Laurencia sp. & $0,12(0,12)$ & $0(0)$ \\
\hline \multicolumn{3}{|l|}{ Corallinales } \\
\hline Corallinaceae (incrustantes) & $0,26(0,17)$ & $4,02(2,25)$ \\
\hline "Algas turfo" (principalmente Corallinaceae articuladas) & $53,48(4,84)$ & $60,94(4,06)$ \\
\hline
\end{tabular}



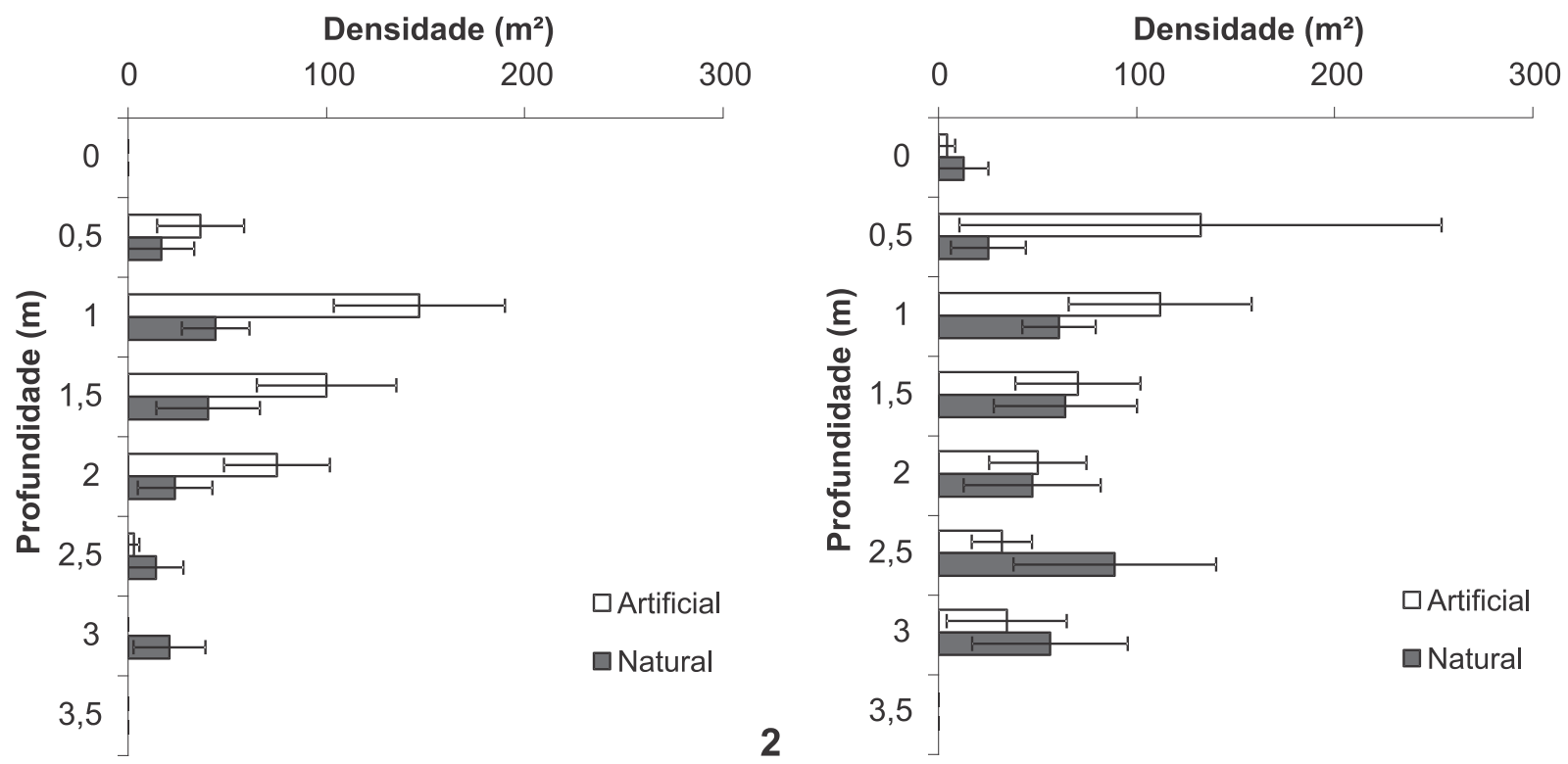

Figs 2, 3. Densidade (média e erro padrão) com profundidade $(\mathrm{cm})$ dos corais invasores em substratos artificiais e naturais na Ilha Grande, estado do Rio de Janeiro, Brasil: 2, Tubastraea coccinea; 3, T. tagusensis.

\section{DISCUSSÃO}

O estudo mostrou que o coral invasor Tubastraea coccinea foi mais abundante em substratos artificiais do que em substratos naturais adjacentes. Em contraste, não foi detectada diferença na abundância de Tubastraea tagusensis em substratos artificiais e naturais. Ainda, a abundância de $T$. coccinea foi relacionada à idade de substrato artificial, o que não foi o verificado com T. tagusensis. As diferenças registradas entre as duas espécies foi devido ao fato que Tubastraea coccinea foi significativamente menos abundante em substratos

Tab. II. Resultados da análise de covariância (ANCOVA) da densidade de Tubastraea coccinea Lesson, 1829 em substratos artificiais e naturais em oito locais com a profundidade como covariável na Ilha Grande, estado do Rio de Janeiro, Brasil.

\begin{tabular}{lcccc}
\hline Fonte de variação & $\begin{array}{c}\text { Graus de } \\
\text { liberdade }\end{array}$ & $\begin{array}{c}\text { Média dos } \\
\text { quadrados }\end{array}$ & $\mathrm{F}$ & $\mathrm{p}$ \\
\hline Profundidade & 1 & 20844 & 1,713 & 0,192 \\
Local & 7 & 55046 & 4,525 & $<0,001$ \\
Substrato & 1 & 77739 & 6,390 & 0,012 \\
Local $\times$ Substrato & 7 & 21682 & 1,782 & 0,091 \\
Erro & 290 & 12166 & & \\
\hline
\end{tabular}

Tab. III. Resultados da análise de covariância (ANCOVA) da densidade de Tubastraea tagusensis Wells, 1982 em substratos artificiais e naturais em oito locais com a profundidade como covariável na Ilha Grande, estado do Rio de Janeiro, Brasil.

\begin{tabular}{lcccc}
\hline Fonte de variação & $\begin{array}{c}\text { Graus de } \\
\text { liberdade }\end{array}$ & $\begin{array}{c}\text { Média dos } \\
\text { quadrados }\end{array}$ & $\mathrm{F}$ & $\mathrm{p}$ \\
\hline Profundidade & 1 & 124283 & 4,606 & 0,033 \\
Local & 7 & 299665 & 11,107 & $<0,001$ \\
Substrato & 1 & 21150 & 784 & 0,377 \\
Local $\times$ Substrato & 7 & 33585 & 1,245 & 0,278 \\
Erro & 290 & 26980 & & \\
\hline
\end{tabular}

naturais (veja Fig. 4). Na mesma região do estudo, CREED \& PAula (2007) relataram que em substratos naturais a densidade de populações adultas de T. tagusensis foi bem maior que de T. coccinea (razão 2:1). Em recrutas a razão foi $3: 2$.

Os resultados aqui apresentados corroboram com a constatação que a modificação do regime de seleção pode ser considerada responsável por explicar a observação de que há correlações entre substratos artificiais e bioinvasão, bem como foi descrito por TYRRELL \& ByERs (2007) para tunicados exóticos em experimentos com placas artificiais e naturais. Apesar disso, CREED $\&$ PAula (2007) relataram que o recrutamento de $T$. tagusensis em cimento foi maior que em azulejos cerâmicos ou placas de aço - estes autores sugerem que este resultado se deve ao fato que cimento é um substrato similar em composição aos recifes de coral (ou seja, quase natural), que é, aparentemente, contraditório com a hipótese de modificação do regime de seleção. Entretanto, o ambiente do píer é bem diferente daquele de placas de recrutamento. Em contraste, T. coccinea

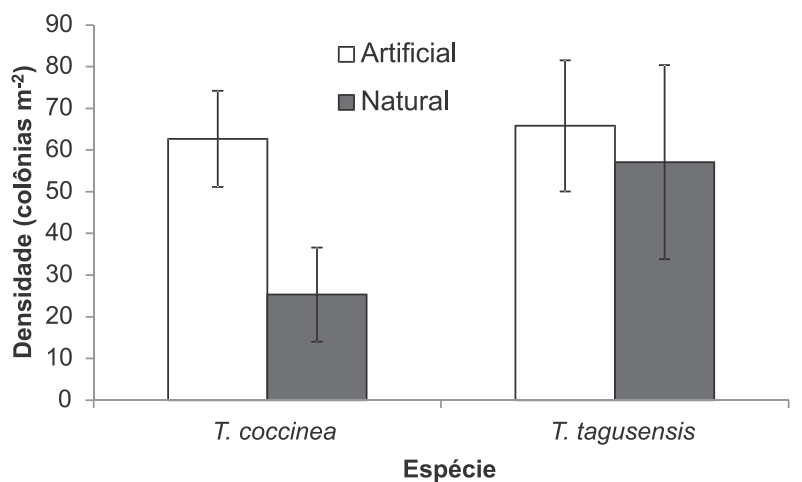

Fig. 4. Densidade (média e erro padrão) de Tubastraea coccinea e $T$. tagusensis em substratos artificiais e naturais na Ilha Grande, estado do Rio de Janeiro, Brasil (barras = erro padrão). 

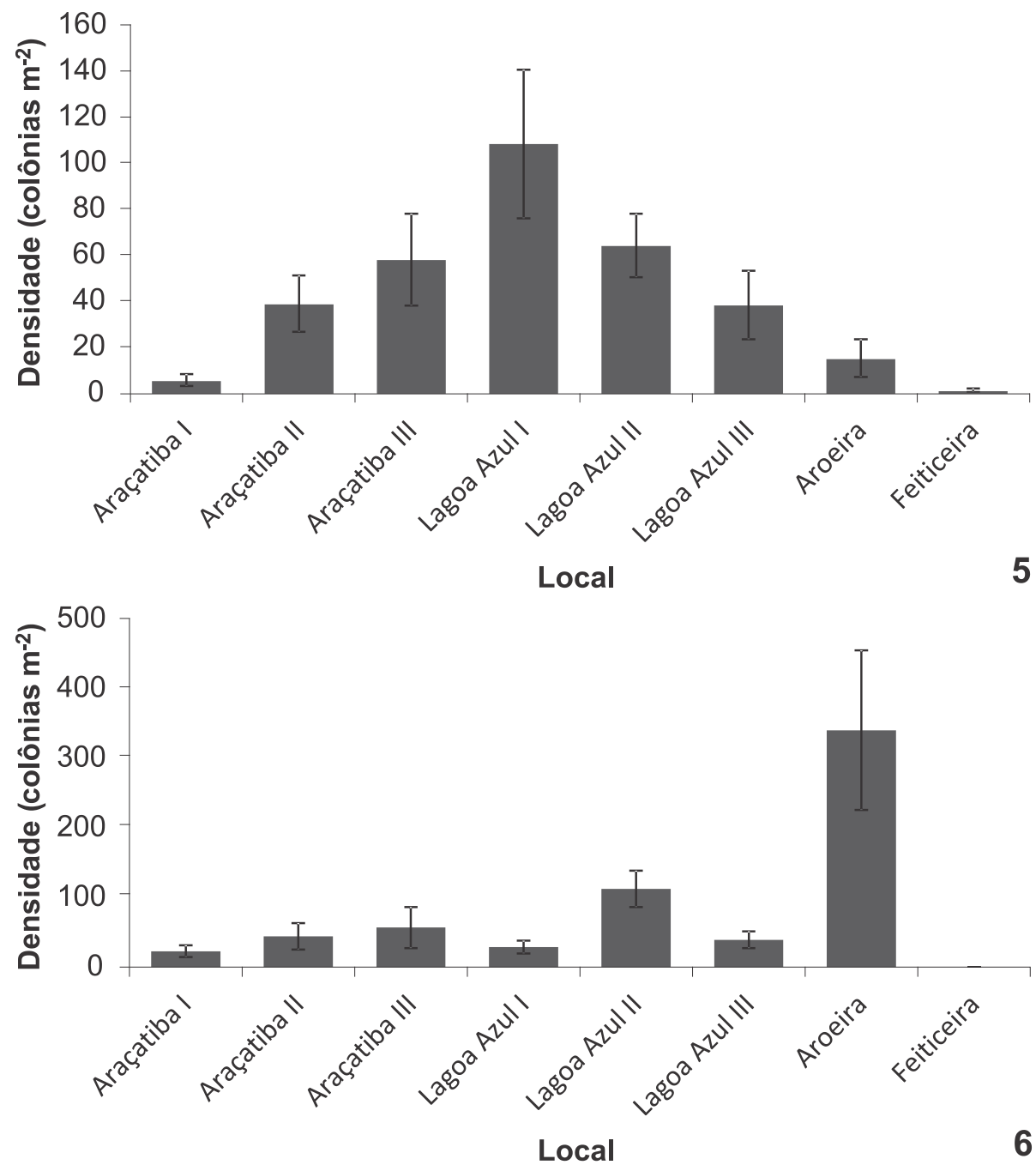

Figs 5, 6. Densidade (média e erro padrão) em oito locais estudados na Ilha Grande, estado do Rio de Janeiro, Brasil: 5, T. coccinea; 6, T. tagusensis.

não mostrou diferença em recrutamento em substratos naturais ou artificiais no mesmo experimento (CREED \& PAula, 2007). Tyrell \& Byers (2007) observaram que em substratos experimentais as espécies exóticas chegaram a ser mais abundantes em placas artificiais paralelamente a uma diminuição nas espécies nativas somente após um período inicial (em que não houve diferença). Talvez os resultados de CREED \& PAulA (2007) se devem ao curto tempo que o experimento foi monitorado (17 meses), comparado a dinâmica de mudança nas comunidades e o tempo de vida de corais. CAngussu et al. (2010) também enfatizam que deve ser levado em consideração o elemento temporal em estudos comparativos de colonização de substratos artificiais e naturais por espécies exóticas.

Tendo em vista a constatação acima e reconhecendo que não há espaço vago nestas comunidades (pois cobertura de biota foi sempre $100 \%$ ), é possível que $T$. tagusensis tem características competitivas superiores e que consegue colonizar substratos artificiais e substratos naturais. De acordo com CREED \& PAULA (2007) as duas espécies têm formas diferentes, pois o coralito de $T$. tagusensis tem projeção maior do coenossarco que $T$. coccinea (18,5 vs. 3,2 $\mathrm{mm}$ respectivamente), que resulta em um crescimento vertical maior em $T$. tagusensis em contraste de $T$. coccinea, que tem um crescimento mais compacto e achatado. A forma de T. tagusensis confere uma vantagem no contexto de competição por espaço com outros organismos sésseis, pois há uma menor probabilidade de mortalidade por recobrimento. LAGES et al. (2010) mencionaram que ambos os corais têm defesas químicas com efeitos negativos sobre elementos da comunidade incrustante e predadores, embora T. tagusensis foi que melhor se defendeu quando comparado com $T$. coccinea em bioensaios in situ. Este conjunto de informações sugere que $T$. tagusensis é competitivamente superior, possuindo vantagens e características que traduzem em uma maior eficiência na inserção em comunidades nativas quando comparada com sua espécie congenérica. Os efeitos de competição interespecífica com a comunidade nativa podem ser tanto positivos quanto negativos para Tubastraea spp. Por exemplo, CREED (2006) constatou que, em 100\% dos contatos com Tubastraea spp., o coral nativo Mussismilia hispida mostrou danos quando em distâncias $\leq 5 \mathrm{~cm}$ das duas espécies invasoras. Entretanto, em outro estudo 


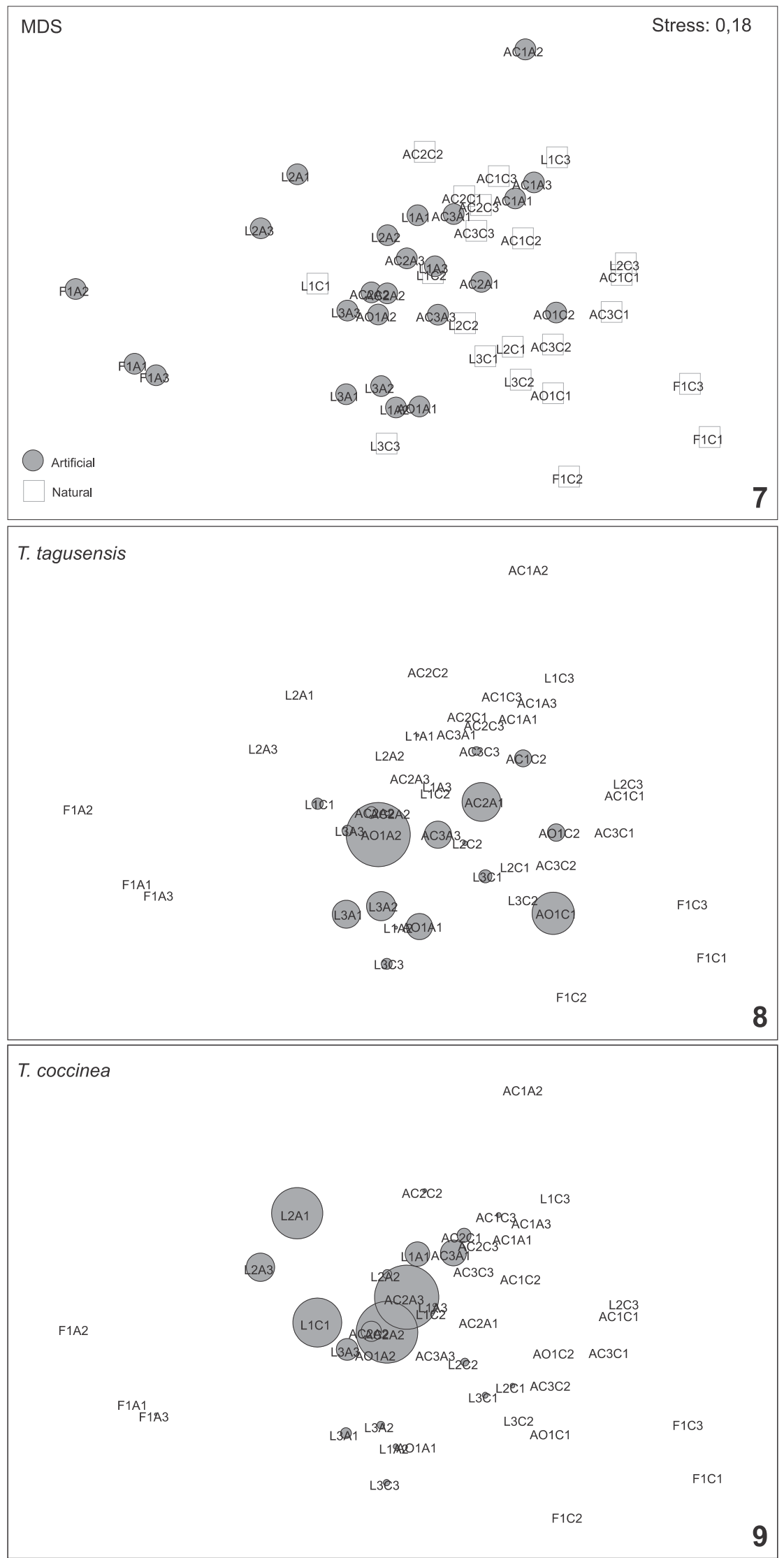

Figs 7-9. Ordenação de comunidades em substratos artificiais e naturais em oito locais na Ilha Grande, estado do Rio de Janeiro, Brasil: 7, Escalonamento Multi-Dimensional (EMD) das comunidades de cada substrato; 8, EDM com superimposição de abundância de T. tagusensis (\% cobertura); 9, EDM com superimposição de abundância de Tubastraea coccinea (\% cobertura) (L, Lagoa Azul; F, Feiticeira; AC, Araçatiba; AO, Aroeira; A, Artificial; C, Natural; o tamanho do círculo cinza é proporcional à abundância do organismo naquela amostra). 
observou-se crescimento da esponja Desmapsamma anchorata Carter, 1882 sobre Tubastraea spp. (MeURer et al., 2010) e não podem ser rejeitados outros processos como recrutamento ou predação diferencial sem estudos experimentais futuros.

Os corais exóticos invasores Tubastraea coccinea e $T$. tagusensis são encontrados nas comunidades biológicas sésseis em costões rochosos da Baía da Ilha Grande, onde são responsáveis por mudanças na estrutura de comunidades locais (LAGES et al. 2011). Foi demonstrado que existem comunidades biológicas distintas em substratos naturais e artificiais: em ambos os substratos houve dominância de tufos algais (53/61\%), esponjas $(7 / 18 \%)$ e cracas $(8 / 10 \%)$. As comunidades se separam devido à presença do zoantídeo Palythoa caribaeorum em substratos naturais (11\%) e abundância maior de Porifera, Caulerpa racemosa (Forsskål) J. Agardh, Carijoa riisei Duchassaing \& Michelotti, 1860, Tubastraea spp. e Schizoporella sp. em substratos artificiais; a presença/ausência de Tubastraea spp. nas comunidades foi importante na ordenação (Figs 8,9 ).

Outros estudos vêm mostrando que Tubastraea spp. têm afinidade com substratos artificiais, especialmente aqueles associados com vetores de transporte. Por exemplo, T. coccinea foi registrada incrustada em plataformas de petróleo e em navios (Fenner, 2001; Fenner \& Banks 2004; Creed \& Paula, 2007; SAMmarco et al., 2010; WANLESS et al., 2010) e hoje é a espécie de coral mais abundante em substratos artificiais no Golfo do México (SAmmarco et al., 2004, 2010). O escleractínio Tubastraea micranthus foi registrado em plataforma de petróleo no Golfo do México, fora de sua área geográfica original (SAMMARCo et al., 2010). PAula \& CReEd (2005) verificaram T. tagusensis em plataformas de petróleo no Brasil.

Em uma abordagem experimental, CREED \& Paula (2007) apontaram elevadas densidades de recrutas de $T$. coccinea e $T$. tagusensis em placas de cimento, granito (natural), azulejo e aço. CREED \& PAUla (2007) concluíram que em substratos de cimento a densidade do Tubastraea tagusensis foi mais elevada do que em cerâmica (azulejos) ou em aço; por outro lado, a densidade do Tubastraea coccinea não mostrou diferença entre os tipos de substratos.

Assim como verificado por PAUla \& CReEd (2005), o presente estudo demonstrou ter encontrado maior abundância de T. tagusensis em profundidades superficiais (rasa), confirmando que a densidade varia de acordo com a profundidade. Organismos bentônicos que se estabelecem em profundidades rasas do infralitoral, por se encontrarem em zona de transição mar-terra, estão sujeitos aos fortes gradientes em fatores ambientais. Aparentemente, T. tagusensis tem habilidade em explorar esses ambientes.

Paula \& CReEd (2005) descreveram que o local com maior densidade de Tubastraea (1132 indivíduos. $\mathrm{m}^{-2}$ ) foi na Lagoa Azul, Ilha Grande. A densidade média de
T. tagusensis encontrada por CREED \& PAULA (2007) em substratos artificiais variou de 202 a 512 colônias $\mathrm{m}^{-2}$ e para T. coccinea entre 187 e 233 colônias $\mathrm{m}^{-2}$; em substratos naturais, a densidade média ( $\pm \mathrm{EP}$ ) de $T$. tagusensis foi de 52,9 colônias $\mathrm{m}^{-2}( \pm 6,5)$ e de $T$. coccinea, 25,5 colônias $\mathrm{m}^{-2}( \pm 4,2)$, sendo também observado no presente estudo maior abundância de T. tagusensis em substratos naturais comparado com $T$. coccinea.

Em relação à idade dos substratos, foi observada uma relação positiva entre a idade (tempo na água) e a abundância de $T$. coccínea; em substratos com idades inferiores a três anos, houve pouca abundância deste invasor. SAMMARCo et al. (2004) relataram que, apesar da abundância total dos corais aumentar significativamente com a idade das plataformas de petróleo no Golfo do México, não houve associação da abundância de $T$. coccinea com a idade do substrato da plataforma, exceto em águas rasas, onde houve relação inversa - diminuição de abundância com o aumento da idade do substrato, que foi interpretado como indicativo de uma espécie pioneira e oportunista. Talvez o período que as estruturas ficaram na água no presente estudo (1-19 anos) foi menor do que as idades de plataformas estudadas por SAMMARCO et al. (2004). CANGuSsu et al. (2010) enfatizaram que tem que ser levado em consideração o elemento temporal em estudos comparativos de colonização de substratos artificiais e naturais por espécies exóticas.

Recentemente, a preocupação com a invasão de espécies exóticas e potencialmente impactantes a comunidades nativas, via bioincrustação, intensificou os levantamentos da biota em regiões próximas aos portos, a fim de detectar a presença de espécies exóticas e/ou potencialmente nocivas que possam ocorrer nas águas que os cercam e, consequentemente mitigar os possíveis danos causados por elas (Procopiak et al., 2006). A introdução de espécies exóticas tais como T. coccinea e T. tagusensis trazem modificações à biodiversidade marinha da região invadida. $\mathrm{O}$ intuito deste trabalho foi investigar se estes invasores tem capacidade de utilizar substratos artificiais disponíveis na região de bioinvasão e comparar tal utilização com substratos naturais locais para entender melhor os processos que estão operando e fornecendo subsídios a futuros estudos ecológicos. A expansão de T. coccinea e T. tagusensis para grande parte dos costões rochosos da Baía da Ilha Grande hoje indica sua alta competitividade e resiliência perante as espécies nativas. Além de estar inserida em comunidades nativas em costões rochosos, $T$. coccinea é mais abundante em pilares de piers, decks ou cais, o que sugere que tais substratos podem facilitar a bioinvasão das comunidades nativas.

Agradecimentos. Ao Projeto Coral-Sol do Instituto Biodiversidade Marinha pelo apoio logístico na realização das etapas de campo; a Petrobras pelo patrocínio através do Programa Petrobras Ambiental; ao coordenador de aquicultura da Prefeitura de Angra 
dos Reis Andre L. Araujo pela atenção e apoio; a Dra. Rosana Rocha pela revisão e comentários em versão anterior do manuscrito. J.C.C. recebeu bolsa do Programa de Incentivo à Produção Científica, Técnica e Artística, UERJ e CNPq, além de auxílios financeiros da FAPERJ $\left(\mathrm{n}^{\circ} \mathrm{E}-26 / 110.857 / 2009\right), \mathrm{CNPq}\left(\mathrm{n}^{\circ} 479000 / 2007-7\right)$ e CAPES (PE Ciências do Mar 1137/2010).

\section{REFERÊNCIAS BIBLIOGRÁFICAS}

Bulleri, F. \& Airoldi, L. 2005. Artificial marine structures facilitate the spread of a nonindigenous green alga, Codium fragile ssp. tomentosoides, in the north Adriatic Sea. Journal of Applied Phycology 42:1063-1072.

Bulleri, F. \& Chapman, M. G. 2004. Intertidal assemblages on artificial and natural habitats in marinas on the northwest coast of Italy. Marine Biology 145:381-391.

BYERs, J. E. 2002. Impact of non-indigenous species on natives enhanced by anthropogenic alteration of selection regimes. Oikos 97:449-458

Cairns, S. D. 1994. Scleractinia of the temperate North Pacific. Smithsonian Contributions Zoology 557:1-150.

2000. A revision of the shallow-water azooxanthellate Scleractinia of the Western Atlantic. Studies of the Natural History of the Caribbean Region 75:1-240

Cangussu, L. C.; Altvater, L.; Haddad, M. A.; Cabral, A. C.; Heyse, L. H. \& Rocha, R. M. 2010. Substrate type as a selective tool against colonization by non-native sessile invertebrates. Brazilian Journal of Oceanography 58:219-231.

Castro, C. B. \& Pires, D. O. 2001. Brazilian Coral Reefs: What we already know and what is still missing. Bulletin of Marine Science 69:357-371.

Creed, J. C. 2006. Two invasive alien azooxanthellate corals, Tubastraea coccinea and Tubastraea tagusensis, dominate the native zooxanthellate Mussismilia hispida in Brazil. Coral Reefs 25:350.

Creed, J. C. \& Oliveira, A. E. S. 2007. Uma metodologia e análise de impactos ambientais. In: Creed, J. C.; Pires, D. O. \& Figueriedo, M. A. O. org. RAP Ilha Grande: um levantamento da biodiversidade. Brasília, DF: MMA/SBF v.1. p. 349-377.

Creed, J. C. \& Paula, A. F. 2007. Substratum preference during recruitment of two invasive alien corals onto shallow-subtidal tropical rocky shores. Marine Ecology Progress Series 330:101-111.

Creed, J. C.; Oliveira, A. E. S. \& De Paula, A. F. 2008. Cnidaria, Scleractinia, Tubastraea coccinea Lesson, 1829 and Tubastraea tagusensis Wells, 1982: Distribution extension. Checklist 4:297300.

ELton, C. S. 1958. The ecology of invasions by animals and plants. London, Methuen \& Co. 196p.

FENNER, D. 2001. Biogeography of three Caribbean corals (Sclaractinia) and the invasion of Tubastraea coccinea into the Gulf of Mexico. Bulletin of Marine Science 69:1175-1189.

FenNeR, D. \& Banks, K. 2004. Orange Cup Coral Tubastraea coccinea invades Florida and the Flower Garden Banks, Northwestern Gulf of Mexico. Coral Reefs 23:505-507.
Ferreira, C. E. L. 2003. Non-indigenous corals at marginal sites. Coral Reefs 22:498

HobBs, R. J. \& HuEnNEKe, L. F. 1992. Disturbance, diversity, and invasion-implications for conservation. Conservation Biology 6:324-337.

Lages, B. G.; Fleury, B. G.; Menegola, C. \& Creed, J. C. 2011 Change in tropical rocky shore communities due to an alien coral invasion. Marine Ecology Progress Series 438:85-96.

Lages, B. G.; Fleury, B. G.; Pinto, A. C. \& Creed, J. C. 2010. Chemical defenses against generalist fish predators and fouling organisms in two invasive ahermatypic corals in the genus Tubastraea. Marine Ecology 31:473-482.

Lira, S. M. A.; Farrapeira, C. M. R.; Amaral, F. M. D. \& Ramos, C. A. C. 2010. Sessile and sedentary macrofauna from the Pirapama Shipwreck, Pernambuco, Brazil. Biota Neotropica 10:155-166

Meurer, B. C.; Lages, N. S.; Pereira, O. A.; Palhano, S. \& Magalhães, G. M. 2010. First record of native species of sponge overgrowing invasive corals Tubastraea coccinea and Tubastraea tagusensis in Brazil. Marine Biodiversity Records 3:1-3.

Paula, A. F. \& Creed, J. C. 2004. Two species of the coral Tubastraea (Cnidaria, Scleractinia) in Brazil: a case of accidental introduction. Bulletin of Marine Science 74:175-183.

2005. Spatial distribution and abundance of nonindigenous coral genus Tubastraea (Cnidaria, Scleractinia) around Ilha Grande, Brasil. Brazilian Journal of Biology 65:661-673.

Procopiak, L. K.; Fernandes, L. F. \& Moreira-Filho, H. 2006. Diatomáceas (Bacillariophyta) marinhas e estuarinas do Paraná Sul do Brasil: lista de espécies com ênfase em espécies nocivas. Biota Neotropica 6:1-28

Sammarco, P. W.; Atchison, A. D. \& Boland, G. S. 2004. Expansion of coral communities within the Northern Gulf of Mexico via offshore oil and gas platforms. Marine Ecology Progress Series 280:129-143.

Sammarco, P. W.; Porter, S. A. \& Cairns, S. D. 2010. A new coral species introduced into the Atlantic Ocean - Tubastraea micranthus (Ehrenberg 1834) (Cnidaria, Anthozoa, Scleractina): an invasive threat? Aquatic Invasions 5:131-140.

Simberloff, V. D. 1997. The biology of invasions. In: Simberloff, V. D.; Schmitz, D. C. \& Brown, T. C. eds. Strangers in Paradise: Impact and Management of Nonindigenous Species in Florida. Island Press, Washington. p. 3-19.

Tyrrell, M. C. \& Byers, J. E. 2007. Do artificial substrates favor nonindiginous fouling species over native species? Journal of Experimental Marine Biology and Ecology 342:54-60

Wanless, R. M.; Scott, S.; SAuer, W. H. H.; AndRew, T. G.; Glass, J. P.; Godfrey, B.; Griffiths, C. \& Yeld, E. 2010. Semi-submersible rigs: a vector transporting entire marine communities around the world. Biological Invasions 12:2573-2583

Wasson, K.; Fenn, K. \& Pearse, J. S. 2005. Habitat differences in marine invasions of central California. Biological Invasions 7:935-948 